\title{
Interstitial Dendritic Cell Guidance by Haptotactic Chemokine Gradients
}

Michele Weber, ${ }_{1}^{1}$ Robert Hauschild, ${ }_{1}^{1}$ Jan Schwarz, ${ }^{1}$ Christine Moussion, ${ }_{1}^{1}$ Ingrid de Vries, ${ }^{1}$ Daniel F. Legler, ${ }^{2}$ Sanjiv A. Luther, ${ }^{3}$ Tobias Bollenbach, ${ }^{1}$ Michael Sixt ${ }^{1 *}$

Directional guidance of cells via gradients of chemokines is considered crucial for embryonic development, cancer dissemination, and immune responses. Nevertheless, the concept still lacks direct experimental confirmation in vivo. Here, we identify endogenous gradients of the chemokine CCL21 within mouse skin and show that they guide dendritic cells toward lymphatic vessels. Quantitative imaging reveals depots of CCL21 within lymphatic endothelial cells and steeply decaying gradients within the perilymphatic interstitium. These gradients match the migratory patterns of the dendritic cells, which directionally approach vessels from a distance of up to 90-micrometers. Interstitial CCL21 is immobilized to heparan sulfates, and its experimental delocalization or swamping the endogenous gradients abolishes directed migration. These findings functionally establish the concept of haptotaxis, directed migration along immobilized gradients, in tissues. 
and the concept that gradients trigger directional migration has not been addressed with manip ulative approaches.

We used mature dendritic cells (DCs) that migrate from the dermal interstitium into affer ent lymphatic vessels (LVs) (II) as a model sys tem to study chemokine function in situ. To track DCs en route to LVs, we used tissue explants of split mouse ears (12). Within minutes after addition of exogenous DCs onto exposed der mal tissue, the cells entered the interstitium and approached nearby LVs in a directed manner (Fig. 1, A to D, and movie S1). Single cell track ing within the almost planar anatomy of the mouse ear (fig. S1) revealed that cells switched from nondirectional to increasingly directional persistent movement toward the vessel at a distance of about $90 \mu \mathrm{m}$ from the vessel wall (Fig. 1, B to D, and fig. S2). When located be tween two LVs, DCs occasionally extended protrusions toward both vessels, indicating the simultaneous presence of two conflicting sig nals. There the cells often spanned distances of more than $50 \mu \mathrm{m}$ before they retracted one protrusion and finally entered one of the vessels (Fig. 1E and movie S2). In line with previous

1IST Austria (Institute of Science and Technology Austria), Am Campus 1, A 3400 Klosterneuburg, Austria. 'Biotechnol ogy Institute Thurgau (BITg) at the University of Konstanz, Unterseestrasse 47, CH 8280 Kreuzlingen, Switzerland. ${ }^{3}$ De partment of Biochemistry, University of Lausanne, Chemin des Boveresses 155, CH 1066 Epalinges, Switzerland.

*To whom correspondence should be addressed. E mail: Sixt@ist.ac.at studies (13), DCs deficient for the chemokine receptor CCR7 did not approach LVs. CCR7 dependency was cell autonomous, because the presence of comigrating wild type DCs could not rescue CCR7 deficient cells (Fig. 1F). Hence, the involvement of secondarily induced paracrine guidance cues that might relay the directional information was excluded. CCR7 has two lig ands, CCL19 and CCL21. By using tissues and DCs from CCL19 deficient animals (14), we found, in agreement with an earlier report (15), that CCL19 was not required for intravasation into afferent LVs (fig. S3).

We therefore measured the localization of the other CCR7 ligand, CCL21, within the der mis. Whole mount immunostainings of ear sheets that were previously fixed and permeabilized re vealed a punctuate CCL21 staining that was exclusively associated with LVs as revealed by lymphatic vessel endothelial hyaluronan receptor 1 (LYVE 1) and basement membrane stainings (Fig. 2, A and B). Disnupting the trans Golgi network by tissue treatment with brefeldin $A$ led to loss of the punctuate pattem and to scattering of CCL21 within individual LYVE 1 bordered (16) lymphatic endothelial cells (Fig. 2B). This suggests that the main source of CCL21 produc tion is lymphatic endothelium, which harbors intracellular depots of this chemokine. Because intracellular CCL21 is not available for cells, es pecially when located at a distance from the ves sel, we used unfixed, nonpermeabilized tissues to exclusively detect extracellular chemokine. The punctuate pattern was not detected. Although the signals were considerably lower than in the per meabilized tissue, the interstitial distribution of CCL 21 became apparent: CCL21 peaked on the vessel wall and appeared to gradually fade with increasing distance from the vessel (Fig. 2C).

To quantitatively analyze these interstitial gradients, we first ensured that our detection method amplified the chemokine signal linearly (fig. S4) and then measured the averaged in tensity of interstitial CCL21 staining as a func tion of distance from the LV margin. Integration of data from multiple ear sheets revealed that the mean CCL21 signal steeply decayed around the vessel and flattened in the intervessel area (Fig. 2D and fig. S5, A and B). Stainings for CCL19 with identical secondary reagents revealed a uniform pattem of background signal in both CCL19 deficient and control tissues. This back ground signal invariably approached the leveled out CCL21 signal remote from the vessel (Fig. 2D and fig. S5, C to G). The slopes of CCL21 gradients appeared smooth upon signal integra tion over large areas or multiple samples. How ever, migrating single cells have only access to local information, which exhibited considerably more noise, with local concentration peaks that could potentially trap the cells on their path to the vessel. The widely accepted spatial para digm of eukaryotic (as opposed to prokaryotic) gradient sensing assumes that cells quantita tively detect concentration differences over their entire surface and polarize toward higher con centrations (17). Hence, we calculated vector maps of the local gradients as they would be
A

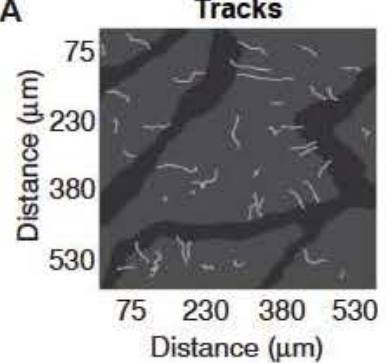

C

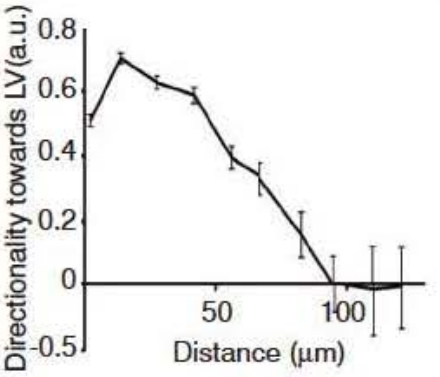

B

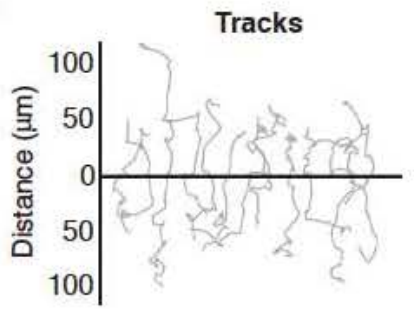

E
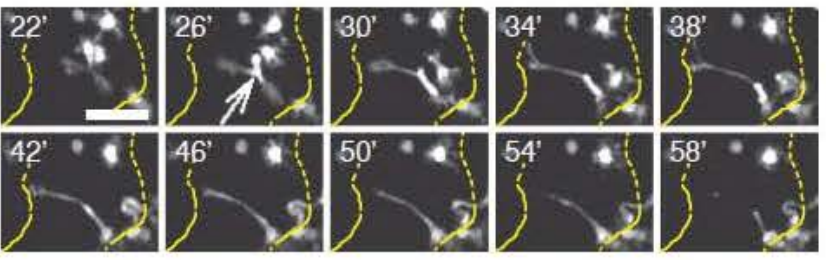

D

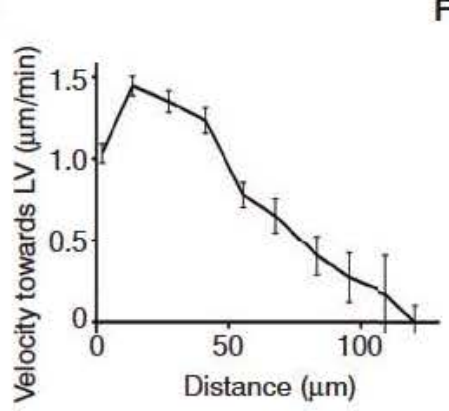

F WT/CCr 7 - DCs

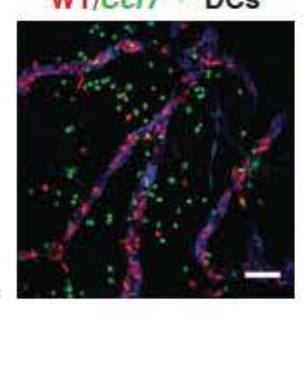

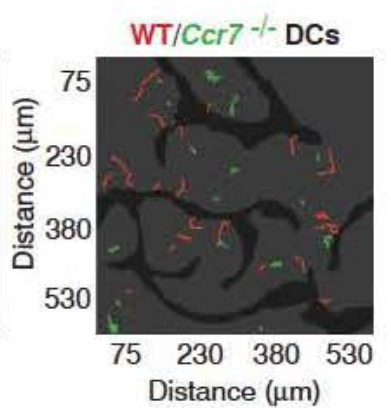

Fig. 1. DCs move directionally toward CCL21-expressing LVs in the dermal interstitium. (A to D) Tracks of DGs migrating in ear explants. (A) Migratory paths (bines in gray) tracked from a representative 60 -min movie, overlaid onto the LV mask (dark gray). (B) Selected tracks (gray) from five movies $(n=$ 200, three independent experiments) reorientated to nearest LV (LV margin at distance $=0$ indicated by the black horizontal line). (C and D) Directionality and velocity toward LV as a function of distance to the nearest LV.
Mean \pm SEM is shown. $n=200$, three independent experiments. a.u., arbitrary units. (E) Wild-type DC (highlighted by the white arrow) migrating between two adjacent vessels (indicated by yellow dotted lines). Scale bar indicates $50 \mu \mathrm{m}$. (F) (Left) Z-stack projection showing wild-type (red) and $\mathrm{CCr} 7^{-1-}$ DCs (green) after 120-min incubation with ear sheets stained for LYVE-1 (blue). Scale bar, $100 \mu \mathrm{m}$. (Right) DC migration paths tracked from a representative 60-min movie, overlaid onto the IV mask (dark gray). 
sensed by a cell of a given diameter. For cell diameters below $9 \mu \mathrm{m}$, no coherent vector field emerged (Fig. 2E and movie S3). When as suming a cell size of 15 to $50 \mu \mathrm{m}$, which reflects the fluctuating span of a migrating DC (Fig. $1 E)$, we retrieved vector fields pointing from the interstitium toward the next vessel for CCL21 but not for control stainings (Fig. 2, E and F, and movie S2). We next computed average local concentration deltas for given cell sizes as a func tion of distance from the vessel. For an assumed cell size of $36 \mu \mathrm{m}$, this "functional gradient"
A

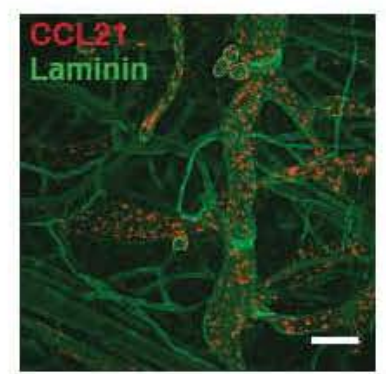

C

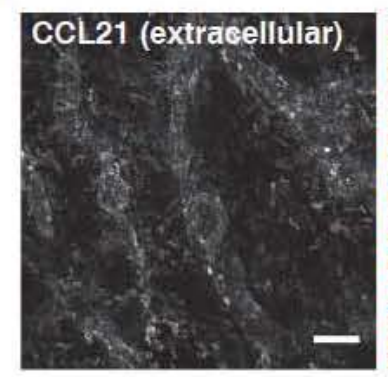

E

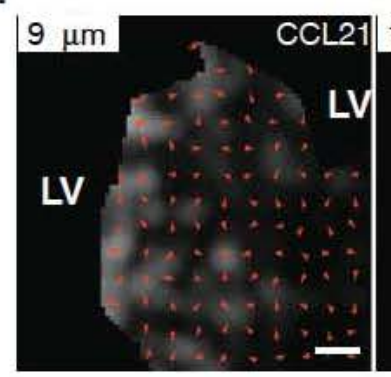

F

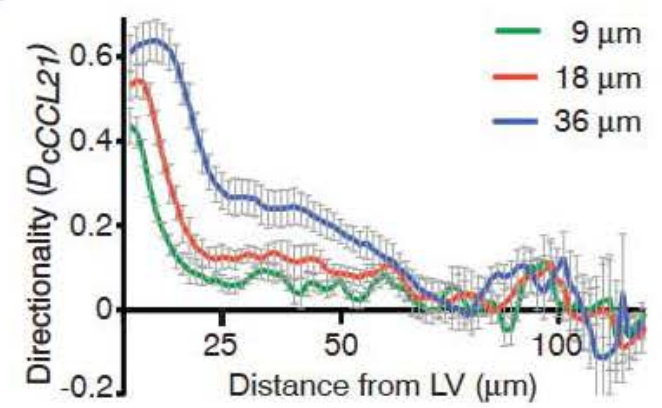

Fig. 2. Visualization and quantification of an interstitial CCL21 gradient. (A) Z-stack projection of permeabilized ear dermis stained for CCL21 (red) and laminin (green). Scale bar, $100 \mu \mathrm{m}$. (B) CCL21 (green) LYVE-1+ (red) costaining of permeabilized ear dermis after treatment with $25 \mu \mathrm{g} / \mathrm{ml}$ brefeldin A. Scale bar, $25 \mu \mathrm{m}$. (C) Z-stack projection of nonpermeabilized ear dermis stained for $\mathrm{CCL}_{21}$. Gray scale shows maximum intensity projection (left). Right image shows same staining as color-coded average projection. LV boundaries are indicated by the blue dotted line based on LYVE-1 staining (as shown in fig. S2). Scale bars, $100 \mu \mathrm{m}$. A representative image from $n=9$ out of four independent experiments is shown. (D) Quantification of interstitial CCL21 and CCL19 staining as function of distance from the nearest LV margin. Mean signal intensities relative to average maximum $C C L 21$ signal \pm SEM are shown (red,
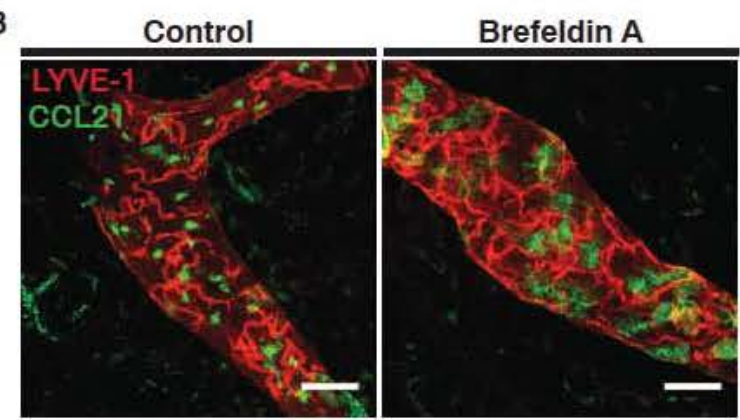

D
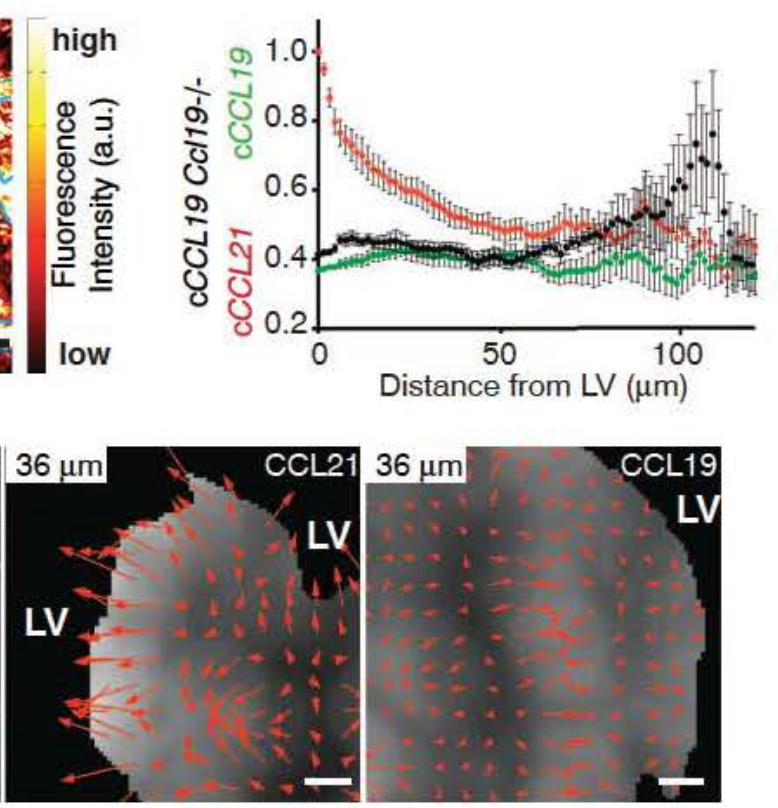

G

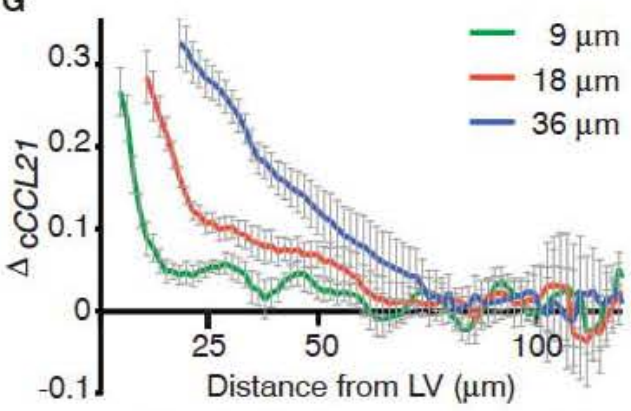

CCL21; green, $\mathrm{CCL} 19$ in $\mathrm{CCl}_{1} 9^{+/+}$ear; $n=9$; four independent experiments; black, CCL19 in $\mathrm{CCl} 19^{-1-}$ ear; $n=5$; two experiments). (E) Vector maps of local chemokine gradients. Arrow length and direction indicate concentration rise in CCL21 after averaging over circular surface area with indicated diameter (virtual cell size). Gray scale indicates averaged intensities. Scale bar, $15 \mu \mathrm{m}$. (F) Directionality of CCL21 gradients $\left(D_{c}\right)$ for indicated virtual cell size as cosine of angle between direction toward closest point of LV and direction of increasing chemokine concentration. Means \pm SEM for $n=6$ from three independent experiments are shown. (G) Average local CCL21 concentration delta $(\Delta)$ as a function of distance to nearest LV, calculated for indicated virtual cell size. Means \pm SEM for $n=7$ from four independent experiments are shown. 
faded at a distance of 75 to $90 \mu \mathrm{m}$ from the vessel wall (Fig. 2G) and thus matched the migratory behavior of the cells that increased directionality in a similar perimeter around the vessel as we have shown above (Fig. 1C). To estimate how well the detected gradients comply with the dis tribution of LVs within the dermis, we calcu lated distance maps of afferent LVs over large areas. We found that a cell that is randomly lo calized within the interstitium would need to travel an average distance of $47 \pm 3 \mu \mathrm{m}$ to reach the nearest LV (fig. S6, A and B) and that $92 \%$ of the interstitial space lies within a $90 \mu \mathrm{m}$ perimeter around the LV network (fig. S6C). In accordance with this, endpoint analysis of in travasation assays only occasionally revealed few cells that were left "stranded" in the middle be tween vessels with large spacing (fig. S6D). These data demonstrate a relative invariability of the intervessel distance and imply that the range of the CCL21 gradient is very well adapted to the distribution of LVs in the skin.

We next challenged our correlative evidence for DC guidance by a CCL21 gradient using experimental manipulations. To test to what de gree a continuous release of CCL21 is required to maintain the gradient, we extensively washed the ear explants and performed migration assays in the presence of brefeldin A to terminate pos sible chemokine secretion from LVs. Because we previously found that CCL21 can be proteolytical ly cleaved at the $\mathrm{C}$ terminus by a $\mathrm{DC}$ associated protease (18), we also pharmacologically blocked this putative cleavage event, which might cause solubilization of CCL21. In both cases, we found that cells still approached LVs (fig. S7, A and B) and conclude that a continuous release of CCL21 is dispensable for directed DC migration within the approximate 2 hour time window of our as says. This suggests that the functionally active CCL21 gradient is stored within the homeostatic dermis.

In contrast to CCL19, CCL21 has a highly charged $\mathrm{C}$ terminal extension that binds glyco saminoglycans (GAGs) and is thought to im mobilize the chemokine to extracellular matrix or cell surfaces $(18,19)$. Our previous findings together with the fact that we could detect CCL21 gradients by histological methods, which necessarily include washing steps where unbound proteins are removed, were consistent with the assumption that CCL21 in the dermis is not sol uble. We therefore performed a series of experi ments in which we perturbed the distribution of endogenous CCL 21 by addition of exogenous chemokine. To this end, we pre incubated ear explants with an excess of exogenous recombi nant CCL21, washed them to remove the solu ble CCL21 fraction, and quantified the impact on gradient shapes and DC migration. We found that exogenous CCL21 diffusely localized with in the dermal interstitium and thereby masked and flattened the endogenous CCL21 gradients (Fig. 3A and fig. S8A). In the pretreated ex plants, a large fraction of DCs was misguided within the interstitium and ultimately remained scattered remote from the vessels (Fig. 3, B to D; fig. S8B; and movie S4). Additionally, pre treatment of the explants with a $\mathrm{C}$ terminally truncated version of CCL21, which is incapable of binding GAGs (19), as well as with a GAG binding but nonsignaling mutant did not change the migratory pattern of the cells (Fig. 3D and fig. S8C). To exclude that exogenous CCL21 af fects DC migration nonspecifically, for example, by masking electrostatic interactions, we pre treated ear explants with exogenous CXCL13 and CXCL12, which also bind GAGs (20) yet

\section{A}

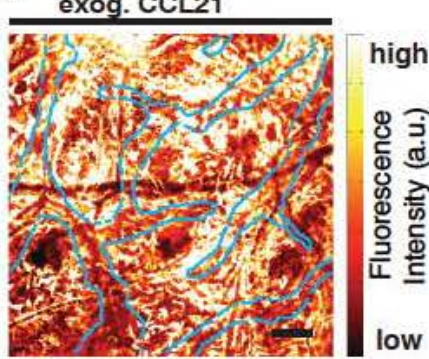

C
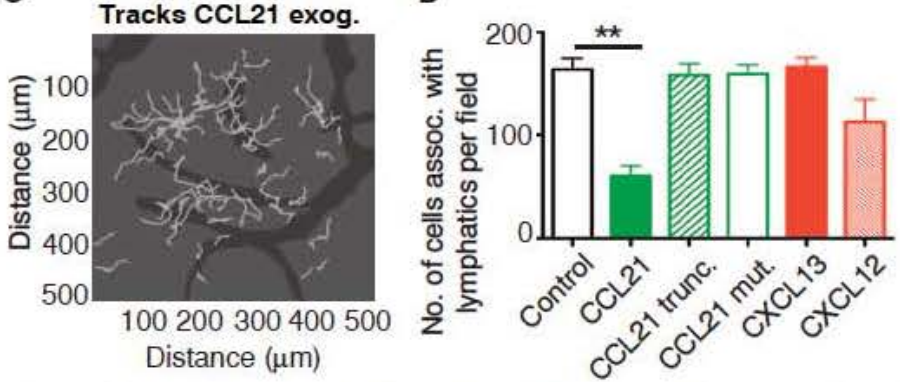

Fig. 3. The CCL21 gradient is functional and immobilized to heparan sulfates. (A) (Left) Z-stack projection of CCL21 immunostaining (false color-coded) of nonpermeabilized ear dermis after incubation with $0.6 \mu \mathrm{g} / \mathrm{ml} \mathrm{CCL21.} \mathrm{LYVE-1-}$ positive LVs are indicated by the blue dotted line. Scale bar, $100 \mu \mathrm{m}$. (Right) CCCL21 after incubation with exogenous CCL21. Gray line indicates endogenous CCL21 as shown in Fig. 2. $n=3$, two independent experiments. (B) Z-stack projections of DCs (red) and LYVE-1 immunostaining (green) after a 120-min co-incubation of DCs with ear sheets. Tissue was either vehicle-treated or incubated with CCL21 before addition of DCs. $n=3$, two independent experiments. (C) DC tracks (light gray lines) from representative 60-min movie with CC21 pre-incubation, overlaid onto LV mask (dark gray). (D) Number of DCs associated with LVs after 120-min crawl-in. Indicated chemokine was preincubated at $0.6 \mu \mathrm{g} / \mathrm{ml}$ before addition of DCs. Control versus CCL21, $* * P=0.006$; control versus CQ21trunc, $P=0.382$; control versus CCL21mut, $P=0.8163$, control versus $X$ XC13, $P=0.458$; control versus CXCL12, $P=0.0642$. $n=3$, at least two independent experiments. (E) Z-stack projection of nonpermeabilized ear dermis stained for CCL21 (false colorcoded, left) and quantification of CCCL21 (right) in nonpermeabilized ear dermis after treatment with 50 mIU heparitinase. Gray line in graph indicates endogenous CCL21 as in Fig. 2. (F) Numbers of DGs associated with LVs in ear dermis after 120 min of crawl-in. $n=5$, three independent experiments ( $\left.{ }^{\star *} P=0.004\right)$. All scale bars, $100 \mu \mathrm{m}$. Error bars indicate SEM. 
do not trigger CCR7, and saw no significant ef fects on DC migration (Fig. 3D and fig. S8C). Together, these findings establish the functional activity of the immobilized CCL21 gradients that we identified before and show that, although DCs carry the receptor for and can respond to CXCL12, CCL21 gradients dominate. In addition, the che mokine pattern appears not to be determined by the distribution of CCL21 binding sites but most likely by the diffusion range of CCL21, which is trapped by sugar residues once it is released from the LVs (21).

CCL21 was shown to bind via its C terminal domain sulfated sugars like heparin, heparan, dermatan, and chondroitin sulfates with low nano molar affinities $(19,22,23)$, potentially explain ing the observed long retention times of CCL21 within the dermis and the inability of $\mathrm{C}$ terminally truncated CCL21 to outcompete the endogenous gradients. To test which of the sugar moieties are involved in the immobilization of CCL21, we pretreated ear explants with sugar degrading enzymes and found that heparitinase, which ef fectively removed heparan sulfates (fig. S9A), severely changed the CCL21 pattern. Quantifica tions revealed an almost complete flattening of the gradient and a drop to signal levels similar to control stainings, whereas tissue integrity was not affected (Fig. 3E and figs. S5G, S9B, and S10A). Consequently, DC migration in heparitinase treated explants was severely diminished (Fig. $3 \mathrm{~F}$ and fig. S10B). Heparan sulfate distribution patterns in untreated dermis did not match these of CCL21 (fig. S9A), corroborating the concept that not the tissue binding sites for CCL21 but rather its distribution range determines the shape of the CCL21 gradient. These findings demon strate that, like in the lumen of blood endothe lium (2), interstitial CCL21 is immobilized to heparan sulfate residues, which either decorate cell surfaces or interstitial matrix components. This immobilized chemokine fraction is sufficient to guide intravasation of DCs, whereas adhesion molecules of the integrin family are dispensable for path finding (24).

The term "haptotaxis" was originally intro duced to describe cell migration along adhesive gradients, a phenomenon that was successfully constituted in vitro but still lacks direct in vivo support (25). Interstitial guidance by heparan sul fate immobilized chemokine gradients, as dem onstrated here, can be viewed as a second variant of haptotaxis. The facts that (i) many chemokines bind GAGs (26), (ii) GAG interaction is impor tant for the leukocyte recruiting activity of some chemokines upon instillation into animals (27), and (iii) leukocytes have the ability to migrate along immobilized chemokine gradients in vitro $(28,29)$ suggest that haptotaxis could be a wide ly used principle. Because immobilized gradients are insensitive to mechanical perturbations, they certainly constitute a robust and stable infrastruc ture for cellular guidance, whereas attraction by soluble gradients might be rather transient in nature.

\section{References and Notes}

1. J. Middleton et al., Cell 91, 385 (1997).

2. X. Bao et al., Immunity 33, 817 (2010).

3. R. Alon, Immunity 33, 654 (2010).

4. E. C. Butcher, L. J. Picker, Science 272, 60 (1996).

5. S. H. Wei, I. Parker, M. ]. Miller, M. D. Cahalan, Immunol. Rev. 195, 136 (2003).

6. F. Castellino et al., Nature 440, 890 (2006).

7. L. I. R. Ehrlich, D. Y. Oh, I. L. Weissman, R. S. Lewis, Immunity 31, 986 (2009).

8. B. Boldajipour et al., Cell 132, 463 (2008).

9. T. Okada et al., PLoS Biol. 3, e150 (2005).

10. B. McDonald et al., Science 330, 362 (2010).

11. D. Alvarez, E. H. Vollmann, U. H. von Andrian, Immunity 29, 325 (2008).

12. H. Pflicke, M. Sixt, J. Exp. Med. 206, 2925 (2009).

13. R. Förster et al., Cell 99, 23 (1999).

14. A. Link et al., Nat. Immunol. 8, 1255 (2007).

15. M. R. Britschgi, S. Favre, S. A. Luther, Eur. J. Immunol. 40, 1266 (2010).

16. P. Baluk et al., J. Exp. Med. 204, 2349 (2007).

17. A. Levchenko, P. A. Iglesias, Biophys. J. 82, 50 (2002)

18. K. Schumann et al. Immunity 32, 703 (2010).

19. ]. Hirose et al., Biochim. Biophys. Acta 1571, 219 (2002).

20. J. L. de Paz et al., ACS Chem. Biol. 2, 735 (2007).

21. P. Müller, A. F. Schier, Dev. Cell 21, 145 (2011).

22. M. Bax, S. J. van Vliet, M. Litjens, J. J. García Vallejo, Y. van Kooyk, PLoS One 4, e6987 (2009).

23. K. Uchimura et al., BMC Biochem. 7, 2 (2006).

24. T. Lämmermann et al., Nature 453, 51 (2008).
25. R. J. Petrie, A. D. Doyle, K. M. Yamada, Nat. Rev. Mol. Cell Biol. 10, 538 (2009).

26. A. Rot, U. H. von Andrian, Annu. Rev. Immunol. 22, 891 (2004).

27. C. L. Salanga, T. M. Handel, Exp. Cell Res. 317, 590 (2011)

28. A. Rot, Eur. J. Immunol. 23, 303 (1993).

29. U. Haessler, M. Pisano, M. Wu, M. A. Swartz, Proc. Natl. Acad. Sci. U.S.A. 108, 5614 (2011).

Acknowledgments: We thank M. Frank for technical assistance and S. Cremer, P. Schmalhorst, and E. Kiermaier for critical reading of the manuscript. This work was supported by a Humboldt Foundation postdoctoral fellowship (to M.W.), the German Research Foundation (Si1323 1,2 to M.S.), the Human Frontier Science Program (HFSP RGP0058/2011 to M.S.), the European Research Council (ERC StG 281556 to M.S.), and the Swiss National Science Foundation (31003A 127474 to D.F.L., 130488 to S.A.L.). The authors declare no conflicts of interest. The data reported in the manuscript are tabulated in the main paper and in the supplementary materials.

\section{Supplementary Materials}

www.sciencemag.org/cgi/content/full/339/6117/328/DC1

Materials and Methods

Figs. S1 to S10

References (30)

Movies S1 to S4 